\title{
Structural Static Characteristic Analysis of Lower Limb Exoskeleton Based on Finite Element Modeling
}

\author{
Liai Pan ${ }^{1, \text { a }}$, Chunshan $\mathrm{He}^{2, \mathrm{~b}}$, Qinghua $\mathrm{Li}^{3, \mathrm{c}}$ \\ ${ }^{1,2,3}$ College of Mechanical and Vehicle Engineering Changchun University, Changchun, 130022 , \\ China \\ aemail: panli636@126.com, bemail: he03cs@126.com, email: zhitu@126.com
}

Keywords: Lower Limb Exoskeleton; Statics Analysis; Finite Element Analysis; ANSYS

\begin{abstract}
Lower limb rehabilitation exoskeletons worn on the outside of the body, which has many features such as wearing comfortable, using safety, efficient rehabilitation training and etc. Structural performance of the exoskeleton determines the overall quality. In the paper, the concepts of the finite element analysis and the theory basis have been introduced. According to ANSYS Workbench, the static characteristic analysis has been carried on in the human body normal gait posture. The analysis results show that the design of the exoskeleton strength stiffness could satisfy the requirements of weight $100 \mathrm{~kg}$ patients using; the stress value and deformation value larger area of the exoskeleton have been achieved. It provided theoretical data for security maintenance of the late exoskeleton.
\end{abstract}

\section{Introduction}

Lower limb rehabilitation exoskeletons worn on the outside of the body, which has many features such as wearing comfortable, using safety, efficient rehabilitation training and etc. Structural performance of the exoskeleton determines the overall quality. Therefore, in the paper, according the ANSYS software, the finite element analysis of the exoskeleton in a human body normal gait has been carried on. Through the static analysis of the exoskeletons, check whether strength and stiffness of the exoskeleton met the design requirements, find, maximum stress and maximum deformation of the exoskeleton in order to prevent the damage of the exoskeleton.

\section{Basic theory of finite element analysis}

Finite Element Analysis (FEA) method, is a continuous integral structure, divided into a limited number of units, at the same time is defined for each unit such as defining unit material properties, some real constant, and other attributes. For each unit is calculated first, and then combine each discrete unit to analyze and calculate, the stress and strain of all nodes and the cell have been achieved, and then solve the conditions of this area generally, the solution problem is obtained. This solution is the approximate solution, but with the increase of number of units and impro1ving accuracy of the interpolation function, ,approximate solution is approaching the real solution [1]. Calculation precision of the finite element method is high, and it can adapt to all kinds of complicated shape, is the effective means of engineering analysis.

The theory bases of finite Element Analysis are the variation principle and elasticity mechanics, involving the basic equation for example equilibrium differential equations, the geometric equations, physical equations in rectangular coordinates, etc.

\section{Static analysis of lower limb exoskeleton}

Static analysis is to calculate the deformation, stress and strain of the structure under fixed load, used to check the strength and stiffness in order to ensure the requirement of the security and stability of the structure. Fixed load includes fixed inertial load, and those load which can be approximately equivalent to the static load which change with time slowly. 


\subsection{D model simplifications of Lower limb rehabilitation exoskeletons}

Whether finite element model is correct or not affects the accuracy of the static analysis results. But for the complex structures, it is very difficult to establish the correct finite element model. Therefore, before finite element analysis model of the exoskeleton was established, model of the exoskeleton should been reasonably simplified.

On the premise of guarantee the force, the part which don't need the analysis should been treated with the large approximate treatment. The exoskeletons have been simplified, the following:

(1) Remove the back frame, motor, reducer transfer auxiliary support system;

(2) Simplify and remove bearings, gaskets, positioning of each joint;

(3) Simplified and remove the screw on the adjustable bar, simplified and deleted holes, chamfering, thread, rounded of the exoskeleton;

(4) When lower limb exoskeletons were simplified, the wear boots have been simplified a whole board.

The lower limb rehabilitation training exoskeleton which has been simplified was shown in figure 1.

\subsection{Grid partition of the lower limb exoskeleton}

After the lower limb rehabilitation exoskeleton three-dimensional model was simplified, the next step was to mesh grip model simplified and generate units. Element meshing quality directly affects the accuracy of analysis results and real credibility, so the meshing is important step in the process of finite element analysis. [2].

ANSYS Workbench has the following several different meshing method: A) automatic classification method; B) tetrahedral partition method; C) scanning method; D) domain method.

Automatic meshing method is to point to in the process of dividing grid, division of grid in the tetrahedron method between scanning type dividing mesh and automatic switch, the switch depends on the geometry can be scanning is divided. For division of grid of lower limb rehabilitation exoskeleton, the automatic classification method was adopted, the grid size was set to $8 \mathrm{~cm}$, and division result of grid of was shown in figure 2.

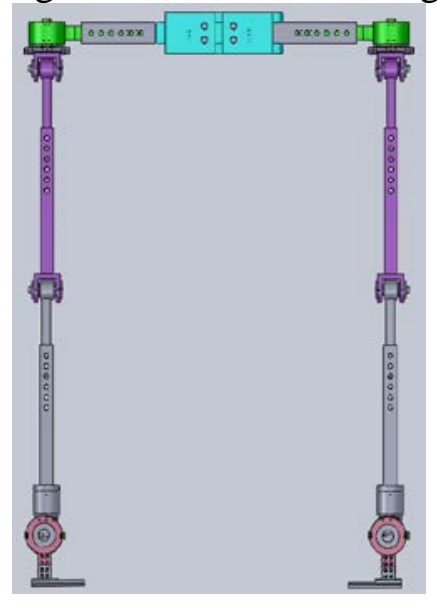

Fig.1. lower limb exoskeleton simplified

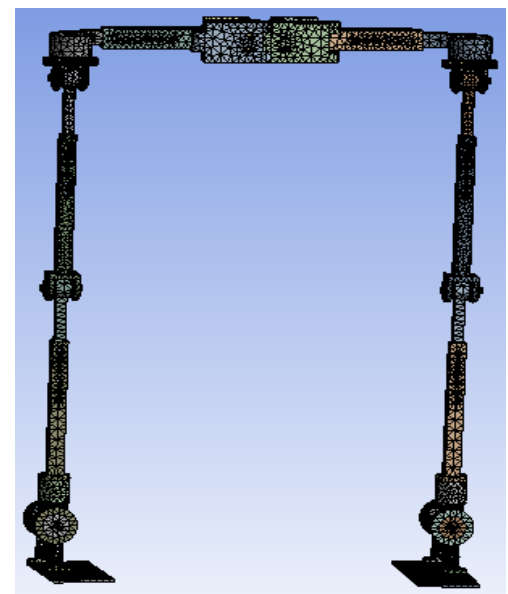

Fig.2. Division of grid of lower limb exoskeleton

\subsection{Create a contact}

Contact is used to simulate the contact, friction and relative sliding between the two parts, is a highly nonlinear problem. It is very important for finite element analysis to create the appropriate contact.

ANSYS Workbench's Mechanical contact provides five different types: Bonded, No Separation, Frictionless, Rough, Frictional, its characteristics are shown in table 1.[3] 
Table.1. Characteristics of different contact types

\begin{tabular}{|c|c|c|c|}
\hline Contact types & Number of iterations & Normal separation & Tangential slip \\
\hline Bonded & once & No clearance & No slip \\
\hline No Separation & once & No clearance & Allowed to slip \\
\hline Frictionless & more than once & permissible clearance & Allowed to slip \\
\hline Rough & more than once & permissible clearance & No slip \\
\hline
\end{tabular}

The advantages of the binding contact as follows:

1) There is no contact convergence problem, solving fast;

2) Because the small deformation only need iterative step a child and a balance, it can be used as a linear analysis to solve

3) Allow large deformation to solve nonlinear analysis.

Because the binding contact has more advantages, in this paper, the binding contact has been used to the contact between components of the lower limb rehabilitation exoskeleton

\subsection{Define the load}

Load of the lower limb rehabilitation exoskeleton was mainly the weight of the human body, motor, battery, quality control system, etc, and its own gravity. When design, with the exception of bone own weight, other these quality should been added together. The biggest total weight was 120 $\mathrm{kg}$. Load loading area is same to gravity location of the human body normal lower limb gait week period, the load was $1200 \mathrm{~N}$ which was the sum of half of human body weight, direction was vertical downward, and vertical to the ground, load figure loaded was shown in figure 3.

\subsection{Constraints loading}

Boundary constraints is an important part of the exoskeleton structure finite element analysis, the appropriate right external skeletal loading boundary constraints, ensure the stiffness matrix non-singular, so as to obtain the exact solution of displacement.

In this paper, exoskeleton was imposed constraints which were fixed constraint of the lower limb exoskeleton, Exoskeleton fixed constraint was same to contact area between the feet and the ground of human lower limb in a normal gait cycle under each position. Exoskeleton constraints were imposed parts was shown in figure 3.[4]



(a) Foot followed to the ground

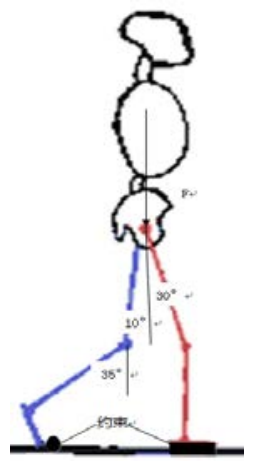

(b) Reaction period of load



(c) Support period

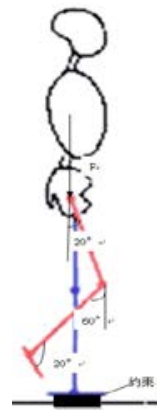

(d) Swing early period

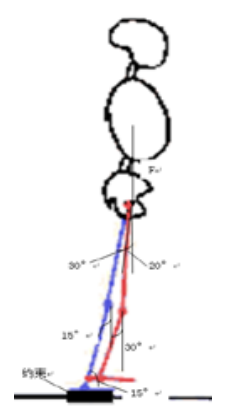

(e) Swinging mid-period

Fig.3. Lower limb constraints load loading 


\section{Results and analysis}

\subsection{Load diagram of each posture putting constraints[5]}

When human lower limb is normally walking, center of gravity of human body is swinging in the frontal plane. So the force applied should coincide with swing position of the center of gravity.

By dynamics analysis of the hip department, in a gait cycle and reaction period, load of hip of support leg was about four times of the body weight. When the exoskeleton was designed, assume half of the total stress strength design exoskeleton, which is $2600 \mathrm{~N}$. In accordance with the design of the total force of $1200 \mathrm{~N}$, the rest force was applied.

When the feet followed the ground, the tiptoe of the left foot and heel strike of the right foot followed to the ground, the vertical force of $1000 \mathrm{~N}$ was on the left and right hip, and the left foot tiptoe and right foot heel in have been applied fixed constraint;

In load reaction period, tiptoe of left foot and palm of right foot were on the ground, constraint was loaded in the left toe and right foot palm, the vertical force of $1600 \mathrm{~N}$ was on the right hip places, and the vertical force of $1000 \mathrm{~N}$ was on the left hip;

In support phase, palm of the right foot was on the ground, left foot was dangling, fixed constraints was imposed on palm of the right foot, the vertical force of $800 \mathrm{~N}$ was on the right hip, and the vertical force of $200 \mathrm{~N}$ was on the left hip;

In early swing phase, palm of the left foot was on the ground, the right foot was impending, fixed constraint was loaded on palm of the left foot, the vertical force of $900 \mathrm{~N}$ was on the left hip, and the vertical force of $300 \mathrm{~N}$ was on the right hip;

In swinging mid-period, palm of the left foot was on the ground, the right foot was impending, fixed constraint was loaded on palm of the left foot, the vertical force of $1000 \mathrm{~N}$ was on the left hip, and the vertical force of $200 \mathrm{~N}$ was on the right hip;

Position in swinging end-period was the same as the foot followed the ground, that is, lower limb movement into the next cycle. Therefore, load and constraints imposed of a gait cycle have been completed in swinging mid-period. Methods of constraint load applied were shown in figure 4.

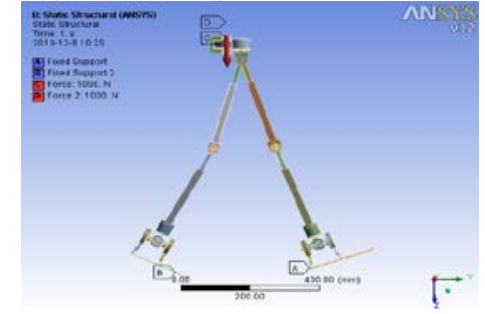

(a) Foot followed to the ground

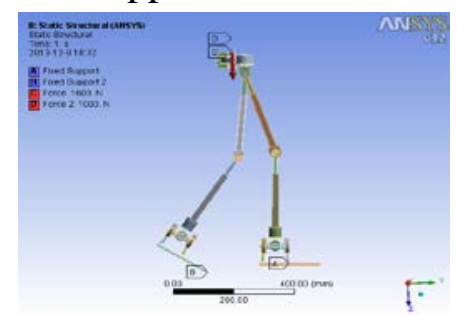

(b) Reaction period of load

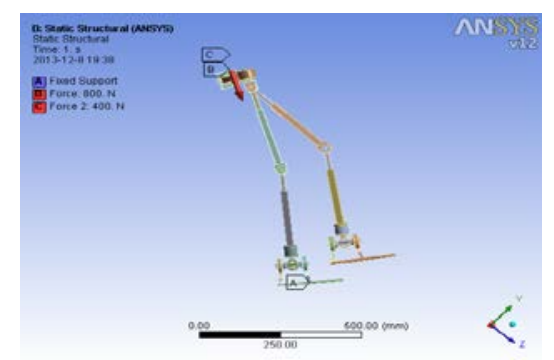

(c) Support period

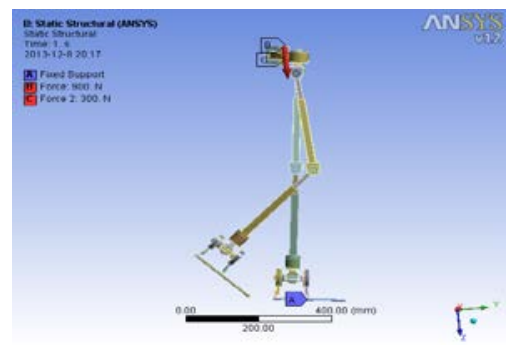

(d) Swing early period



(e) Swinging mid-period

Fig.4. Constraint load applied figure of positions of the exoskeleton

\subsection{Static analysis results [6]}

After the completion of the applied load, constraints and analysis, stress diagrams of the lower limb rehabilitation exoskeletons have been achieved, as shown in figure 5. 


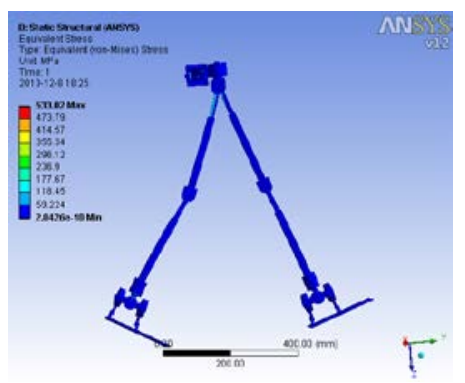

(a) Foot followed to the ground

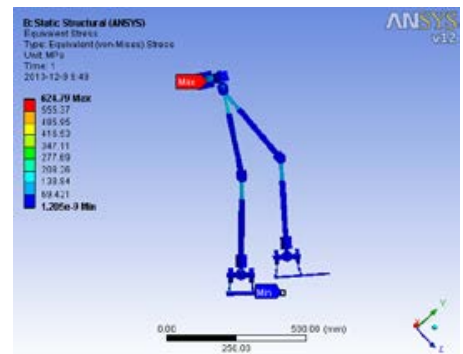

(c) Support period

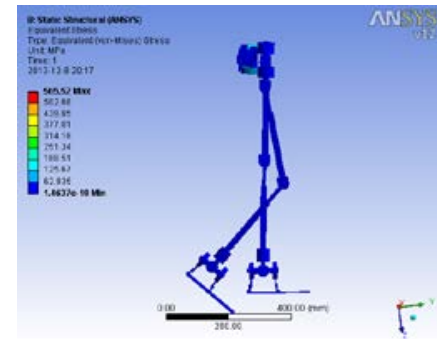

(d) Swing early period

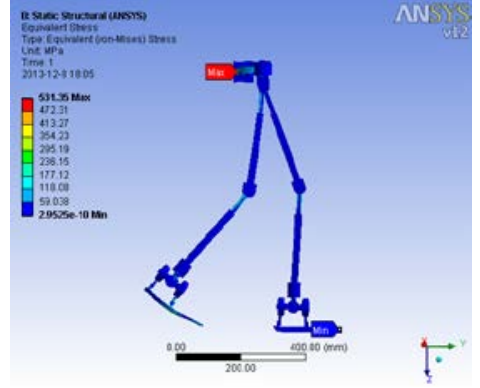

(b) Reaction period of load

Fig.5. Stress diagrams of the lower limb rehabilitation exoskeletons

Elastic strain diagrams of static characteristic analyses of Lower limb rehabilitation exoskeleton were shown in figure 6.

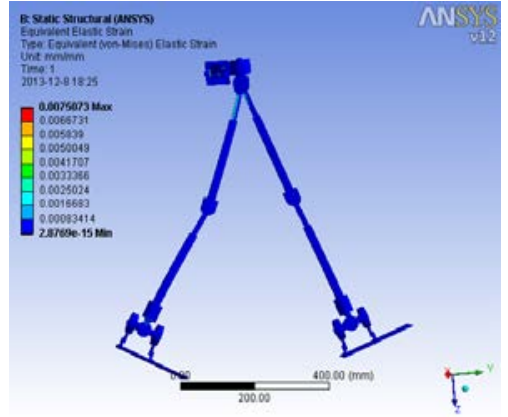

(a) Foot followed to the ground

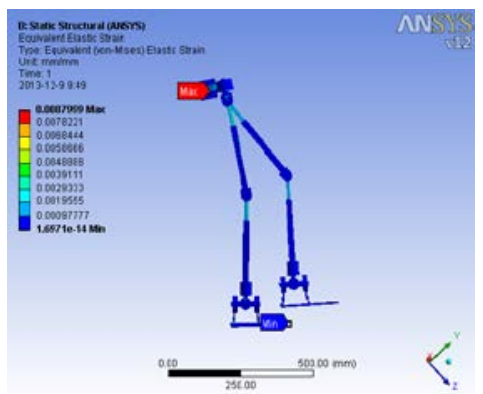

(c) Support period

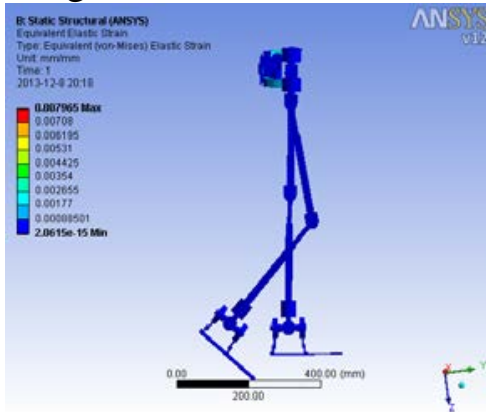

(d) Swing early period

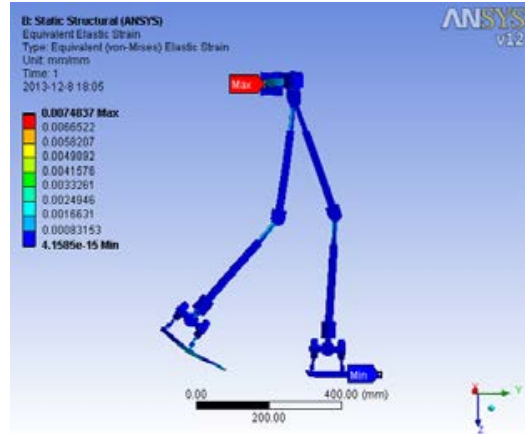

(b) Reaction period of load

Fig.6. Elastic strain diagrams of lower limb rehabilitation exoskeleton

The figures show that The maximum stress value of the lower limb exoskeleton structure was $624.79 \mathrm{MPa}$, which appeared in supporting the right hip; the maximum deformation of the lower limb exoskeleton was $0.0087999 \mathrm{~mm}$ in supporting the right hip; stress distribution of the lower limb exoskeleton structure was more homogeneous, mainly concentrated in between 65-260 MPa.

Hip joint of the lower limb exoskeleton was a major. In the moment position changing, the force of the hip joint was all bigger. But the maximum stress and maximum deformation were in the range of material allowable values, thus, stiffness and strength of this exoskeleton structure all met 
the design requirements.

\section{Conclusion}

In the paper, the concepts of the finite element analysis and the theory basis have been introduced. According to ANSYS Workbench, the static characteristic analysis has been carried on in the human body normal gait posture. The analysis results show that the design of the exoskeleton strength stiffness could satisfy the requirements of weight $100 \mathrm{~kg}$ patients using; the stress value and deformation value larger area of the exoskeleton have been achieved. It provided theoretical data for security maintenance of the late exoskeleton.

\section{References}

[1] Yuejin Shang. Principle of finite element and ANSYS application guide[M].Beijing. Tsinghua University Press, 2005

[2] Guangyi Pu. ANSYS Workbench 12 basic tutorials and example explanation[M].Beijing: China WaterPower Press, 2010

[3] Xiaohua Zhu, Zhixiang Yu,etc. Finite element analysis of ANSYS advanced engineering examples chosen[M]. Beijing: Electronic Industry Press, 2004

[4] A.Mohsenimanesh,et al Stress analysis of a multi-laminated tractor tyre using non-linear 3D finite element analysis[J].Materials and Design,2009,30:1124-1132

[5] A.C.Seibi,M.F.Amateau,Finite element modelling and optimization for controlling the residual thermal stresses of laminated composite tubes[J].Composite Structures,1998,(41)2: 151-157

[6] Jialong Wu. Elastic mechanics[M]. Beijing: Higher Education Press, 2001 